\title{
\begin{tabular}{llllllllll}
$\mathrm{C}$ & $\mathrm{O}$ & $\mathrm{M}$ & $\mathrm{M}$ & $\mathrm{E}$ & $\mathrm{N}$ & $\mathrm{T}$ & $\mathrm{A}$ & $\mathrm{R}$ & $\mathrm{Y}$ \\
\hline
\end{tabular}
}

\section{Proposed Air Quality Objectives for Fine Particulate Air Pollution}

\author{
Tee L. Guidotti
}

Canada is now considering recommendations of the Federal-Provincial Working Group on Air Quality Objectives and Guidelines to adopt objectives for fine particulates that will be among the most stringent anywhere in the world and that may be technically unobtainable in the short term. No Canadian city would be in compliance at this time if these objectives were adopted.

The fine particulate issue is also driving a fundamental change in the way that Canada harmonizes regulations on air pollution. In the United States, standards are set by the federal government for implementation by the states; the federal government has primacy. These standards are mandatory as a minimum level of control, although states may impose more stringent standards. By contrast, in the Canadian system the federal government does not have primacy and environmental protection at the local level is mandated to provincial governments. Canada has chosen to set guidelines negotiated between the federal government and representatives of provincial governments, in the expectation that these guidelines will influence the individual provinces to adopt similar standards. The role of guidelines, as opposed to standards, is to drive voluntary change and to influence public opinion. In the past, these guidelines were set to be more stringent than U.S. National Ambient Air Quality Standards and reflected the reality that urban air pollution levels in most large Canadian cities were generally lower than their U.S. counterparts.

Department of Public Health Sciences, University of Alberta, Edmonton, AB

Correspondence: Dr. Tee Guidotti, Department of Environmental and Occupational Health, School of Public Health and Health Services, The George Washington University Medical Center, $2300 \mathrm{~K}$ Street, NW, Suite 201, Washington, DC 20036
In 1997, a new process was initiated to bring the development of recommended air quality criteria into a more scientific formulation. National Ambient Air Quality Objectives will now be developed in a more transparent, accountable and science-based process. They will also be recommended rather than mandated but as objectives will carry the expectation that all jurisdictions in Canada will eventually harmonize their own standards to these norms.

The first two objectives under this new process are fine particulates and ozone. For the purpose of this discussion, we shall confine our remarks to fine particulates. A large body of evidence was compiled into a report $^{1}$ that constituted the briefing document for a Stakeholder Consultation Workshop convened by the Atmospheric Environment Service of Environment Canada in December 1997. Included in this dossier was the report of the Federal Provincial Working Group recommending new air quality objectives for fine particulates. $^{2}$

The implication of these recommendations was staggering. Based on their reading of the available evidence, and driven primarily by estimates of lives saved through reductions in mortality associated with fine particulate levels in urban areas, the Federal Provincial Working Group recommended an objective for $\mathrm{PM}_{10}$ of a 24hour average concentration of $40 \mu \mathrm{g} / \mathrm{m}^{3}$ and for $\mathrm{PM}_{2.5}$ of $20 \mu \mathrm{g} / \mathrm{m}^{3}$. This compares with the U.S. Environmental Protection Agency's (EPA's) previously proposed (now remanded) National Ambient Air Quality Standard of U.S. 24-hour averages of 150 and $65 \mu \mathrm{g} / \mathrm{m}^{3}$, respectively. Canada would have no equivalent to the previously proposed U.S. annual averages of 50 and $15 \mu \mathrm{g} / \mathrm{m}^{3}$, respectively, relying on the 24 hour average to drive reductions.
These objectives were criticized at the time of the workshop as unobtainable in practice, particularly because much air pollution in Canada is transboundary and therefore out of the control of local authorities. However, the desirability of reducing the risk of Canadians of exposure to fine particulate air pollution and the observation that there is a linear, non-threshold effect does argue for control to be as tight as feasible. The economic implications are great, however, and the proposed objectives must be able to withstand scrutiny if they are to be adopted as a national policy. This means that the risk associated with fine particulates must be validated, the protection to human health offered by reducing exposure should be confirmed and the cost of doing all this should be acceptable.

In retrospect, it is remarkable that fine particulates should play such a strong role as a determinant of human mortality as is suggested by the epidemiological evidence. The effect on health is certainly disproportionate to the tiny mass of the particles in this previously unappreciated size distribution. The absence of a "smoking gun" by which the damage caused by fine particulate matter could be explained has proven to be a major obstacle in air quality studies and air pollution toxicology. The identification of clinicopathological correlations of PM-related health effects has been a major missing link in the evidentiary chain from population health studies to policy implications. The limits of the population-based approach in isolation may have been reached, particularly with respect to $\mathrm{PM}_{2.5}$. Indeed, a minority opinion, even in epidemiology, is that if no medical condition can be clearly identified to be associated with PM-associated risk, the basic criteria for concluding causation, and therefore the rationale for regulation, is lacking. 


\section{TABLE I}

\section{Applying the Hill Criteria for Strength of Epidemiological Evidence for Causal Association to the Association Between Exposure to Fine Particulate Air Pollution and Acute Mortality, on Knowledge Base Available in Spring 1998.}

\section{Hill Criteria}

1. Strength of the association

2. Consistency among studies

on

4. Temporality (exposure must precede outcome)

5. Exposure-response relationship evident

6. Plausibility of mechanism

7. Coherence (chain of evidence)

8. Experimental replication

9. Analogy (a weak criterion)
Arguments Pro

Relationship of $1.5 \% / 10 \mu \mathrm{PM}_{2.5}$ is not a particularly strong association

Numerous studies demonstrate similar association although studies vary by

- Geographic location

- Time period

- Pollutant mix

Total mortality largely reflects cardiopulmonary causes.

This is the essence of time series analysis.

Clearly demonstrated for $\mathrm{PM}_{10^{\prime}}$ further strengthened for $\mathrm{PM}_{2.5}$, suggesting $\mathrm{PM}_{2,5}$ is causal.

Emerging evidence suggests cardiac effects (Godleski). Speculative pathophysiology.

Premature. Too much remains to be learned.

Just beginning to identify likely animal models.

Premature. Pathophysiology not understood.

\section{Arguments Con}

Relative risk of mortality associated with air pollution is typically on

order of $5 \%$; attributable risk is very high because of large populations.

Relatively little variation in method; studies are all conducted along similar lines.

No specific disease entity can be identified in association with exposure.

Not applicable.

Mass of fine particulates is exceedingly small; relevant metric (count, mass) is debatable.

Effects suggested or demonstrated to date are not proportionate to human outcomes.

Premature.

No defined human outcome recognized on which to model.

No obvious analogy exists in toxicology; requires assumptions of great potency for fine particulates.
Satisfied.

Not satisfied.

$\therefore$ Is Criterion Satisfied?

Not satisfied.

Satisfied.

Not satisfied.

Satisfied.

Not satisfied.

Not satisfied.

Not satisfied.
Table I reviews available evidence as it pertains to $\mathrm{PM}_{2.5}$ against the Hill criteria for the acceptance of an epidemiological association to be causal. These criteria are generally accepted for the evaluation of epidemiological evidence as suggestive of causation $^{3}$, although they are subject to their own limitations $s^{4}$. They are intended to be rigorously applied as a set of criteria when the body of evidence is primarily statistical and they may be supplanted as a judgement for causation by mechanistic evidence. Of the nine criteria, only temporality, consistency, and dose-response (on a population basis) are now met given the current body of evidence. However, there is certainly sufficient evidence to accept an association as a working hypothesis for public health intervention.

Alternative explanations for the discrepancy in the Hill criteria include the early stage of knowledge regarding fine particulates and the possible presence of confounding factors that create a spurious association. It is certainly the case that investigation in progressing rapidly and much remains to be learned. However, a diligent search for confounding factors on the part of numerous teams of investigators has yet to suggest a strong candidate. The progressive strengthening of the association with better measurement of particulates, from TSP to $\mathrm{PM}_{10}$ to $\mathrm{PM}_{2.5}$, also argues against a confounder at work.

Progress in defining acceptable levels of ambient air pollutants will require mechanistic as well as population-based research and broader thinking in the analysis of available data. To evaluate the degree of risk in association with specific subpopulations that may be more vulnerable than the general population will require identification of these groups and some notion of the pathology involved. Leading candidates for such susceptibility states may include medical conditions such as congestive heart failure, acute medical conditions such as lower respiratory tract infections, and episodic conditions that involve decompensation, such as asthma. Proof or even strong evidence is lacking, however.

We suggest that a group or panel of physicians, epidemiologists, toxicologists and other biomedical lscientists is needed to review the available evidence and to conduct a gaps analysis on a consensus basis to identify missing elements of data and opportunities for targeted research relevant to air quality in Canada.

The context for setting guidelines urgently requires innovative approaches. One such innovation has been the suggestion of "riskbased" objectives or standards. The observation that there is no threshold for fine particulate effects, but an association with mortality that is more or less linear over the entire range, has raised the question of what risk is "acceptable" and how this might be determined. This is a problem unique to the current situation, where health risk levels are low in absolute terms but there are still public health gains to be made by further reductions. 
These features of the problem suggest the design of an open, social process applied to a defined population at emissions levels below those associated with extreme effects or transboundary damage. This approach may be seen as counter to an emerging trend to base standards on cost/benefit analyses. It would have the advantage of empowering communities to decide for themselves what is an acceptable risk. The key, it is believed, may be to conduct the process on the model of risk comparison rather than a model of quantitative risk assessment or cost/benefit. This approach puts guideline formation on a scientific and social grounding quite different from standards setting and to conform to the particular demographic and air quality situation of Canada.

The Supreme Court of the United States remanded the EPA's proposed rules for both fine particulate air pollution and for ozone, on grounds that the agency had not demonstrated in the rule its mandate to institute such far-reaching regulation without Congressional approval. This development does not change the underlying policy issues with respect to regulating air quality, but it does put Canada in the position of being alone in North America in proposing a very stringent objective that would be particularly sensitive to shortterm changes in air quality.

Canadian regulatory policy needs to decide whether exposure to fine particulates is a public health emergency or a public health opportunity, and whether compliance with these objectives is a national priority or a national preference. The alternatives to adopting these objectives and enforcing standards based on them include a policy of continuous improvement (reflecting changes in control technology, turnover in vehicle fleet and improvements in fuel economy), a risk comparison and prioritization based on public input or a systematic determination of acceptability of risk, as outlined in this paper. There are alternatives to mandated standards for lowrisk environmental hazards.

\section{REFERENCES}

1. Atmospheric Environment Service, Environment Canada. National Ambient Air Quality Objectives: Stakeholder Consultation Workshop, 1 - 2 December 1997, Downsview, Ontario. Environment Canada, Downsview, Ontario, 1997.

2. Federal - Provincial Working Group on Air Quality Objectives and Guidelines. National Ambient Air Quality Objectives for Particulate Matter. Part 2. Recommended Air Quality Objectives. Ottawa, Federal - Provincial Working Group on Air Quality Objectives and Guidelines, October 1997.

3. Hill AB. Environment and disease: association or causation? Proc Roy Soc Med 1965; 58:295-300.

4. Guidotti TL. Hidden assumptions in environmental research. Environ Res 1992; 59:101-13.

\section{E. SASKatOON $\overline{\text { DISTRICT }}$ HE A L T H \\ Deputy MeDical Health Officer Public Health Services}

Saskatoon District Health - Public Health Services is a dynamic and innovative health unit leading the way in the development of a modern public health service infrastructure. We provide a stimulating environment in which multidisciplinary teams provide outcome-based services to meet client and population needs. Services are provided in urban and rural settings with linkages to other health-related agencies in the Saskatoon Health District and the partner districts of Central Plains, Gabriel Springs and Living Sky. The population served is approximately 265,000. The position is based in Saskatoon, a beautiful city with a major university and all social amenities making for excellent family living. Working benefits are very competitive.

Reporting to the Medical Health Officer/General Manager, the new Deputy Medical Health Officer will be part of the team responsible for the management of district public health services.

Candidates must be eligible for registration with the College of Physicians and Surgeons of Saskatchewan and possess a fellowship in community medicine or a post-graduate degree in public health. Supervisory and management experience in community health services and an ability to work co-operatively in a team setting will be essential.

TO APPLY: Please submit your resume in confidence to Brenda Solheim (Phone 306-655-2428; Fax 306-655-2444) at Employment Services, Saskatoon District Health, 103 Hospital Drive, Saskatoon, Saskatchewan, Canada, S7N 0W8 by August 31, 1999. Please quote competition number 9900-0688-20C.

\section{Visit our web site at www.sdh.sk.ca.}

Saskatoon District Health is committed to a representative workforce of Aboriginal people, candidates must self declare in writing that they are a member of the designated group.

Saskatoon District Health thanks all applicants, but only those chosen for interviews will be contacted.

\section{WORKING TOGETHER TO IMPROVE HEALTH}

